\title{
Assistência de enfermagem às fases do transplante renal: uma revisão integrativa
}

\author{
Thaynara Gabriella Silva Cunha ${ }^{1}$ \\ Karine Cardoso Lemos²
}

\begin{abstract}
${ }^{1}$ Enfermeira pela Escola Superior em Ciências da Saúde. Residente em Nefrologia pela ESCS/FEPECS/SES-DF
2 Mestre em Enfermagem pela Universidade de Brasília - UnB. Tutora do Programa de Residência Multiprofissional em Nefrologia da Escola Superior de Ciências da Saúde ESCS/FEPECS/SES. Docente ESCS/FEPECS. Enfermeira SES/DF
\end{abstract}

E-mail para correspondência: thaygabi9@gmail.com

\section{RESUMO}

Introdução: A Doença Renal Crônica (DRC) se configura como importante problema de saúde pública em constante crescimento. Dentre as modalidades de Terapia Renal Substitutiva (TRS) está o Transplante Renal (TR), que vem apresentando maior sucesso em decorrência do desenvolvimento no âmbito técnico-científico, porém ainda há taxas elevadas de rejeição e complicação. É necessária então a atuação da enfermagem para redução destes índices e sucesso do enxerto. Objetivo: Apresentar a assistência da enfermagem nas diferentes fases do transplante renal e sua importância na redução da taxa de rejeição e intercorrências. Método: Trata-se de um artigo de revisão integrativa realizado exclusivamente com base em referências secundárias. Resultados: A amostra final desta revisão foi constituída por 10 artigos científicos cuja análise levou a decisão de realizar apresentação das seguintes temáticas: perfil sociodemográfico, pré-transplante real, transoperatório e pós-operatório. Conclusão: A DRC é um problema de saúde pública em crescimento, assim como a realização do transplante renal caracterizado por uma alta demanda e baixa oferta. O profissional de enfermagem precisa prestar assistência qualificada durante todo o processo para intensificar a sobrevida do enxerto junto ao paciente.

Palavras-chave: Insuficiência Renal Crônica, Transplante de Rim, Cuidados de Enfermagem

Nursing care for kidney transplantation phases: an integrative review

\begin{abstract}
Introduction: Chronic Kidney Disease (CKD) is an important public health problem that is constantly growing. Among the modalities of Renal Replacement Therapy (RRT) is the Kidney Transplant (TR), which has been showing greater success due to the development in the technical-scientific scope, but there are still high rates of rejection and complication. It is necessary, therefore, the performance of nursing to reduce these rates and graft success. Objective: To present nursing care in the different stages of kidney transplantation and its importance in reducing the rate of rejection and complications. Method: This is an integrative review article carried out exclusively based on secondary references. Results: The final sample of this review consisted of 10 scientific articles whose analysis led to the decision to present the following themes: sociodemographic profile, actual pre-transplant, trans-operative and postoperative. Conclusion: CKD is a growing public health problem, as well as kidney transplantation, characterized by high demand and low supply. The nursing professional needs to provide qualified assistance throughout the process to intensify graft survival with the patient.
\end{abstract} Keywords: Chronic Kidney Failure, Kidney Transplantation, Nursing Care. 


\section{INTRODUÇÃO}

A Doença Renal Crônica (DRC) é definida por diagnóstico sindrômico com perda progressiva e irreversível da filtração glomerular e/ou lesões no parênquima renal em período de três ou mais meses ${ }^{1}$.

Configura-se como importante problema de saúde pública mundial que atinge mais de 90 mil pessoas no Brasil, com maior incidência na faixa etária de 19 a 64 anos. Essa taxa tende a aumentar visto que as doenças crônicas vêm afetando a população em escala crescente, devido ao envelhecimento populacional e demora no diagnóstico da condição ${ }^{1}$.

O manejo desta patologia se baseia em terapia farmacológica, nutrição equilibrada, diálise e, quando possível, o transplante renal. A escolha da terapia de substituição renal depende da doença de base, estágio, velocidade de progressão e comorbidades ${ }^{1}$.

As modalidades de terapia renal substitutiva (TRS) incluem a hemodiálise, diálise peritoneal e transplante renal. Em 2016 a Sociedade Brasileira de Nefrologia (SBN) indica que aproximadamente 122 mil pessoas estavam em tratamento dialítico no Brasil. De acordo com o Registro Brasileiro de Transplantes (RBT) foram realizados, em 2016, 5.648 transplantes renais ${ }^{2}$.

Em 2017, a fila de espera pelo transplante renal aumentou de 29.268 (2016) para 31.266, segundo o censo de diálise da Sociedade Brasileira de Nefrologia ${ }^{2}$.

Estudos apontam que o transplante renal é a opção mais completa de substituição renal e proporciona melhor qualidade de vida, por compensar ou substituir o desempenho do órgão sem a necessidade de terapias dialíticas. Possibilita a reinserção do indivíduo no convívio da sociedade e a libertação da diálise, já que é um recurso terapêutico durável, que melhora a sobrevida e oferece uma ótima relação custo-efetividade. A média de custo do transplante é dez 
vezes menor quando comparado à hemodiálise, incluindo os gastos com a terapia imunossupressora ${ }^{1,3,4,5}$.

É indicado para doentes renais crônicos em estágio cinco, mas em algumas situações o transplante preemptivo pode ser considerado, significa a realização do procedimento antes do início da terapia dialítica. Pode ser feito a partir de um órgão sadio proveniente de um doador vivo ou falecido ${ }^{4}$.

Nos últimos anos houve avanços importantes e contínuos na área do transplante renal, com aprimoramento das terapias imunossupressoras, introdução de novos medicamentos, aperfeiçoamento técnico-científico, educação continuada da equipe multidisciplinar, acesso a educação e informação ${ }^{3,5}$.

Porém tem acessibilidade reduzida, já que a demanda cresce a cada dia e o número de rins disponíveis é insuficiente ${ }^{4}$. É necessário ainda haver compatibilidade do sistema ABO, antígenos de histocompatibilidade leucocitária (HLA) e a prova cruzada (cross-match) e, por fim, ainda há risco de haver rejeição do órgão, evoluindo novamente para disfunção renal ${ }^{1,5}$.

Pela complexidade do procedimento cirúrgico de médio porte, em que ocorre ablação de um órgão de uma pessoa e implantação em outra, o paciente pós-transplante renal necessita de suporte emocional e psicoterápico para adaptação e preparo para reestruturação da vida sociofamiliar e sociolaborativa, demandas sociais para adaptação ao novo cotidiano após alta hospitalar e econômicas para arcar com as novas despesas inerentes ao tratamento ${ }^{1,4,5}$.

No momento pós-operatório o paciente também precisa de uma assistência à saúde direta e contínua com monitorização dos parâmetros das funções hepática, renal, hematológica, bioquímica do sangue, o rastreamento de sinais flogísticos, o uso contínuo de medicamentos e os ajustes da prescrição imunossupressora ${ }^{1}$. 
Neste cenário, o enfermeiro pode oferecer uma assistência integral fundamentada no processo de enfermagem através do conhecimento técnico-científico para a tomada de decisão, que constitui instrumento e ferramenta norteadora da sistematização da assistência de enfermagem (SAE) e segue uma teoria de enfermagem, pois desse modo confere mais segurança e qualidade na atuação, além de contribuir para a redução do risco de rejeição e para atingir os objetivos terapêuticos ${ }^{1,5}$.

Este profissional necessita de conhecimento especializado para reduzir, prevenir e antecipar as intercorrências e complicações, além de intervir de imediato para intensificar as chances de sucesso do enxerto em longo prazo, bem como fornecer atenção de qualidade durante todo o processo de internação ${ }^{5}$.

O enfermeiro está em contato direto com o paciente e, por isso, pode contribuir significativamente para a manutenção da saúde e sucesso do transplante. Para isso é fundamental ampliar os conhecimentos para atuar em todo o processo, desde o diagnóstico de morte encefálica do doador, cuidados e viabilidade de seus órgãos na captação, correta abordagem familiar, bem como prover a assistência de alto nível tanto aos candidatos e receptores, quanto aos cuidadores e familiares, de modo a permitir a continuidade da assistência até o trans e pós-trasplante ${ }^{3,4}$.

O desenvolvimento da comunicação terapêutica como estratégia educativa entre equipe e paciente tem sido reconhecida como ferramenta para proporcionar o cuidado integral ${ }^{3}$. É fundamental que o enfermeiro, junto ao paciente, família e equipe multidisciplinar, precisa estar sempre alerta aos fatores de risco e relacionados no contexto dos processos infecciosos para planejar e implementar intervenções direcionadas avaliando sempre a evolução de cada caso.

Apesar dos avanços técnico-científicos na área do transplante renal, as complicações e intercorrências associadas a este procedimento cirúrgico invasivo ainda são frequentes, já que 
a compatibilidade de todos os sistemas envolvidos é um estado difícil de encontrar, considerando ainda a discrepância entre demanda e órgãos disponíveis para doação.

Esta pesquisa foi elaborada pela seguinte questão norteadora: Qual abordagem da assistência de enfermagem, ao paciente transplantado renal em suas três fases, é apresentada nos estudos sobre o tema?

A motivação para a realização deste artigo de revisão integrativa reside na importância da atuação do profissional enfermeiro frente ao sucesso do transplante renal e redução da rejeição do enxerto, visto que este profissional está em contato direto e contínuo com o paciente, assim como está envolvido em todas as etapas do processo, pré, trans e pós- transplante, fator que possibilita detecção precoce de problemas e rápida intervenção, além da sua assistência na educação em saúde de toda a estrutura social e de apoio referente ao indivíduo intensificando assim a chance de sucesso com a implementação de cuidados e estímulo do autocuidado.

\section{OBJETIVO}

Frente ao exposto e buscando estabelecer uma estrutura e destaque da importância da enfermagem no cenário do transplante renal, o presente artigo tem como objetivo apresentar a assistência da enfermagem nas diferentes fases do transplante renal e sua importância na redução da taxa de rejeição e intercorrências.

\section{MÉTODO}

Trata-se de um artigo de revisão integrativa com coleta de dados realizada exclusivamente a partir de fontes secundárias, através de levantamento bibliográfico e baseado na formação em curso da autora pelo Programa de Residência Multiprofissional em Nefrologia da Fundação de Ensino e Pesquisa de Ciências da Saúde - FEPECS da Secretaria de Saúde do Distrito Federal - SES/DF. 
O artigo foi desenvolvido através do levantamento de artigos na literatura com busca nas seguintes bases de dados: Scientific Eletronic Library Online (Scielo), Literatura LatinoAmericana e do Caribe em Ciências da Saúde (LILACS), Biblioteca Virtual de Saúde (BVS) e Medical Literature Analysis and Retrieval System online (Medline).

Foram utilizados, para a busca dos artigos, os seguintes descritores e suas combinações na língua portuguesa e inglesa: "enfermagem" AND "assistência de enfermagem" AND "transplante renal".

Os critérios de inclusão definidos para a seleção dos artigos foram: artigos publicados em português, inglês e espanhol; artigos na íntegra que retratassem a temática referente à assistência de enfermagem no transplante renal e artigos publicados e indexados nos referidos bancos de dados nos últimos 10 anos.

Os critérios de exclusão incluídos no processo de seleção dos artigos foram: artigos publicados em outros idiomas que não fossem português, inglês e espanhol, artigos incompletos, referentes a outros aspectos do transplante fora o assunto abordado, artigos mais antigos que 10 anos.

A análise e a síntese dos dados extraídos dos artigos foram realizadas de forma descritiva, o que permite observar, contar, descrever e classificar os dados, com o intuito de reunir o conhecimento desenvolvido sobre o tema explorado.

\section{RESULTADOS E DISCUSSÃO}

A amostra final desta revisão foi constituída por 13 artigos científicos de um total de 300 artigos, selecionados pelos critérios de inclusão previamente estabelecidos. A tabela 1 representa as especificações de cada um dos artigos. 
Tabela 1 - Artigos levantados nas bases de dados Scielo, BVS, Medline e LILACS sobre assistência de enfermagem no transplante renal.

\begin{tabular}{|c|c|c|c|}
\hline Autor & Título do Artigo & $\begin{array}{l}\text { Periódico (vol., } \\
\text { nº, pág., ano) }\end{array}$ & Considerações/Temática \\
\hline $\begin{array}{l}\text { Albuquerque JG, } \\
\text { Lira ALBC, Lopes } \\
\text { MVO }\end{array}$ & $\begin{array}{llr}\text { Fatores } & \text { preditivos } & \text { de } \\
\text { diagnóstico de enfermagem em } \\
\text { pacientes submetidos ao } \\
\text { transplante renal. }\end{array}$ & $\begin{array}{l}\text { Rev Bras Enferm. } \\
63(1): 98-103 \text {; jan-fev } \\
2010\end{array}$ & $\begin{array}{l}\text { Identifica fatores preditivos de } \\
\text { diagnósticos de enfermagem de } \\
\text { pacientes transplantados renais. }\end{array}$ \\
\hline $\begin{array}{l}\text { Lira ALBC, Lopes } \\
\text { MVO }\end{array}$ & $\begin{array}{l}\text { Pacientes } \text { transplantados } \\
\text { renais: análise de associação } \\
\text { dos diagnósticos de } \\
\text { enfermagem. }\end{array}$ & $\begin{array}{l}\text { Rev Gaúcha Enferm. } \\
\text { 31(1): 108-14; mar } \\
2010\end{array}$ & $\begin{array}{l}\text { Analisa associações entre os } \\
\text { diagnósticos de enfermagem, } \\
\text { fatores relacionados e } \\
\text { características definidoras em } \\
\text { pacientes transplantados renais. }\end{array}$ \\
\hline $\begin{array}{l}\text { Goi C, Meier AC, } \\
\text { Salamoni H, } \\
\text { Zanella MC, } \\
\text { Rosanelli CLSP }\end{array}$ & $\begin{array}{l}\text { Sistematização da assistência } \\
\text { de enfermagem a pacientes } \\
\text { pós-transplante renal }\end{array}$ & $\begin{array}{l}\text { XXI Seminário de } \\
\text { Iniciação Científica; } \\
2013\end{array}$ & $\begin{array}{l}\text { Define SAE e Processo de } \\
\text { Enfermagem, descrevendo suas } \\
\text { etapas aplicadas ao transplante } \\
\text { renal. }\end{array}$ \\
\hline $\begin{array}{l}\text { Inácio LA, } \\
\text { Montezeli JH, } \\
\text { Sade PMC, } \\
\text { Caveião C, Hey } \\
\text { AP }\end{array}$ & $\begin{array}{l}\text { Atuação do enfermeiro nas } \\
\text { orientações de alta ao paciente } \\
\text { pós-transplante renal }\end{array}$ & $\begin{array}{l}\text { Rev Enferm UFSM. } 4 \\
\text { (2): } 323-331 \text {; abr/jun } \\
2014\end{array}$ & $\begin{array}{l}\text { Descreve as orientações de alta } \\
\text { pelo enfermeiro ao paciente pós- } \\
\text { transplante renal. }\end{array}$ \\
\hline $\begin{array}{l}\text { Silva AES, Pontes } \\
\text { UO, Genzini T, } \\
\text { Prado PR, Amaral } \\
\text { TLM }\end{array}$ & $\begin{array}{l}\text { Revisão integrativa sobre o } \\
\text { papel do enfermeiro no pós- } \\
\text { transplante renal }\end{array}$ & $\begin{array}{lr}\text { Cogitare } & \text { Enferm. } \\
\text { 19(3): } & 593-603 ; \\
\text { jul/set } 2014 & \end{array}$ & \begin{tabular}{l}
\multicolumn{3}{l}{ Apresenta o papel do enfermeiro } \\
no pós-transplante renal e \\
evidencia os principais \\
cuidados.
\end{tabular} \\
\hline $\begin{array}{lr}\text { Santos } & \text { CM, } \\
\text { Kirchmaier } & \text { FM, } \\
\text { Silveira } & \text { WJ, } \\
\text { Arreguy-Sena C } \\
\end{array}$ & $\begin{array}{l}\text { Percepções de enfermeiros e } \\
\text { clientes sobre cuidados de } \\
\text { enfermagem no transplante de } \\
\text { rim }\end{array}$ & $\begin{array}{l}\text { Acta Paul Enferm. } \\
\text { 28(4): } 337-43 ; \\
\text { fev/mar 2015. }\end{array}$ & $\begin{array}{l}\text { Analisa as percepções de } \\
\text { enfermeiros e transplantados } \\
\text { sobre a consulta de enfermagem } \\
\text { pré-transplante renal. }\end{array}$ \\
\hline $\begin{array}{l}\text { Câmara JJC, } \\
\text { Queiroz PL, Sousa } \\
\text { SMA, Paiva SS }\end{array}$ & $\begin{array}{l}\text { Estratégias implementadas } \\
\text { pelo enfermeiro para } \\
\text { aprendizagem do transplantado } \\
\text { renal em imunossupressão }\end{array}$ & $\begin{array}{l}\text { Cienc Cuid Saude. } \\
\text { 15(2): } 282-287 ; \\
\text { abr/jun } 2016 .\end{array}$ & $\begin{array}{l}\text { Descreve a vivência da } \\
\text { enfermagem na implementação } \\
\text { de estratégias de aprendizagem } \\
\text { em imunossupressão para } \\
\text { transplantados renais. }\end{array}$ \\
\hline $\begin{array}{lr}\text { Souza } & \text { TL, } \\
\text { Trindade } & \text { TRO, } \\
\text { Mendonça } & \text { AEO, } \\
\text { Silva RAR } & \end{array}$ & $\begin{array}{l}\text { Necessidades humanas básicas } \\
\text { alteradas em pacientes pós- } \\
\text { transplante renal: estudo } \\
\text { transversal }\end{array}$ & $\begin{array}{l}\text { Online Braz J Nurs. } \\
\text { 15(2): 265-275; jun } \\
2016 .\end{array}$ & $\begin{array}{l}\text { Identifica as necessidades } \\
\text { humanas básicas alteradas nos } \\
\text { pacientes que realizaram } \\
\text { transplante renal com base na } \\
\text { Teoria das Necessidades } \\
\text { Humanas Básicas. }\end{array}$ \\
\hline $\begin{array}{l}\text { Primo HFBC, } \\
\text { Hayakawa LW }\end{array}$ & $\begin{array}{l}\text { Conhecimento da equipe de } \\
\text { enfermagem na assistência ao } \\
\text { paciente em pós-operatório de } \\
\text { transplante renal }\end{array}$ & $\begin{array}{l}\text { Rev UNINGÁ } \\
\text { Review. 29(3) } \\
\text { 11-17; } \\
\text { jan/mar 2017. }\end{array}$ & $\begin{array}{l}\text { Investiga o conhecimento da } \\
\text { equipe de enfermagem na } \\
\text { assistência ao paciente no } \\
\text { período pós-transplante renal. }\end{array}$ \\
\hline $\begin{array}{l}\text { Pedro LS, Batista } \\
\text { AP }\end{array}$ & $\begin{array}{l}\text { Assistência de enfermagem ao } \\
\text { paciente submetido a } \\
\text { transplante renal: uma revisão } \\
\text { integrativa }\end{array}$ & $\begin{array}{l}\text { R Enferm UFJF. 3(1) } \\
\text { 51-56; jan/jun } 2017 .\end{array}$ & $\begin{array}{l}\text { Elucida a participação de } \\
\text { enfermagem durante as fases do } \\
\text { transplante renal, com ênfase na } \\
\text { participação do enfermeiro e } \\
\text { revela a importância da sua } \\
\text { atuação. }\end{array}$ \\
\hline $\begin{array}{lr}\text { Dâmaso } & \text { AG, } \\
\text { Santos } & \mathrm{CS}, \\
\text { Bezerra } & \text { ASCE }\end{array}$ & $\begin{array}{l}\text { Assistência de enfermagem } \\
\text { nos cuidados perioperatórios } \\
\text { de pacientes em transplante } \\
\text { renal }\end{array}$ & $\begin{array}{l}\text { Ciências Biológicas e } \\
\text { de Saúde Unit. 4(2) } \\
\text { 271-282; Nov 2017. }\end{array}$ & $\begin{array}{l}\text { Identifica a assistência de } \\
\text { enfermagem nos cuidados } \\
\text { perioperatórios de pacientes em } \\
\text { transplante renal. }\end{array}$ \\
\hline $\begin{array}{lr}\text { Pedroso } & \text { VSM, } \\
\text { Thurow } & \text { MRB, } \\
\text { Medeiro } & \text { AC, } \\
\end{array}$ & $\begin{array}{l}\text { Orientações do enfermeiro e } \\
\text { mudanças no comportamento: }\end{array}$ & $\begin{array}{l}\text { Rev Norte Mineira de } \\
\text { Enferm.8(1) 92-102; } \\
2019\end{array}$ & $\begin{array}{l}\text { Analisa as orientações do } \\
\text { enfermeiro ao usuário de } \\
\text { transplante renal e averigua as }\end{array}$ \\
\hline
\end{tabular}




\begin{tabular}{|l|l|l|l|}
\hline $\begin{array}{l}\text { Scarton J, } \\
\text { Rodrigues ST, } \\
\text { Siqueira HCH. }\end{array}$ & $\begin{array}{l}\text { caminho para a sobrevivência } \\
\text { do usuário transplantado renal }\end{array}$ & $\begin{array}{l}\text { mudanças no } \\
\text { comportamento no } \\
\text { domiciliar, no pós-transplante. }\end{array}$ \\
\hline $\begin{array}{l}\text { Kochhann DS, } \\
\text { Figueiredo AEPL }\end{array}$ & $\begin{array}{l}\text { Enfermagem no transplante } \\
\text { renal: comparação da demanda } \\
\text { de cuidado entre escalas }\end{array}$ & $\begin{array}{l}\text { Acta Paul Enferm. 33: } \\
1-8.2020 .\end{array}$ & $\begin{array}{l}\text { Compara o número de horas da } \\
\text { demanda de cuidados de } \\
\text { enfermagem ao paciente no pós- } \\
\text { operatório de transplante renal. }\end{array}$ \\
\hline
\end{tabular}

$\mathrm{Na}$ análise dos artigos selecionados e evidências científicas encontradas, considerou-se relevante para a pesquisa a apresentação das seguintes temáticas: perfil sociodemográfico, prétransplante real, transoperatório e pós-operatório.

\section{Perfil Sociodemográfico}

Estudos apontam que a maioria dos pacientes transplantados renais é do gênero masculino ${ }^{1,6,7}$. Com idade média de 41 anos, escolaridade com maior prevalência apenas com ensino fundamental seguido de ensino médio ${ }^{6,7}$.

A pesquisa de Albuquerque et al. (2010) evidenciou que os pacientes transplantados no hospital público não trabalham, não produzem, não pagam impostos e não contribuem para a previdência social. Observou também que adolescentes transplantados tendem a interromper os estudos em decorrência das sessões de hemodiálise, e até mesmo após a cirurgia, a recuperação, os cuidados e o acompanhamento ambulatorial limitam o indivíduo quanto ao retorno aos estudos. Isso justifica a baixa escolaridade apresentada ${ }^{7}$.

Souza et al. (2016) encontraram a média de idade de 30 - 40 anos, maioria casados, aposentados ou com auxílio-doença, católicos, com tempo médio de espera para o transplante entre 6 e 10 anos ${ }^{1}$. Maioria significativa (90\%) recebeu o órgão de doador falecido ${ }^{1}$.

\section{Pré-Transplante Renal}

O enfermeiro atua ativamente no processo da doação e captação do órgão, de modo que ao se referir ao possível doador, com diagnóstico de morte encefálica, são atribuições deste profissional: providenciar a declaração de óbito, notificar a equipe, providenciar o quanto antes o processo de constatação de morte encefálica, identificar o possível doador, realizar a 
notificação da equipe de captação de órgão intra-hospitalar e assistir aos familiares de maneira humanizada e transparente. Também é papel fundamental do enfermeiro preservar as condições clínicas e hemodinâmicas do potencial doador. Através desta assistência qualificada que os familiares poderão autorizar a doação ${ }^{8}$.

A atuação do enfermeiro e educação continuada da equipe são instrumentos imprescindíveis para detectar e solicitar as intervenções apropriadas, e se conforma como elemento chave para a viabilização e obtenção do órgão ${ }^{8}$.

A fase de pré-transplante constitui um momento rico e capaz de possibilitar abordagens educativas guiadas pela consulta de enfermagem, esclarecimento de dúvidas, redução de ansiedade e reafirmação de comportamentos de adesão à terapêutica na fase do pós-transplante, ou seja, componentes que refletem área de atuação do enfermeiro e mostram-se essenciais para assegurar o sucesso do procedimento operatório ${ }^{4,8}$.

É papel privativo do enfermeiro aplicar a Sistematização da Assistência de Enfermagem (SAE) com o intuito de prevenir eventos adversos, além disso a visita pré-operatória de enfermagem é reconhecidamente essencial e está inserida em um momento decisivo ${ }^{8}$.

\section{Transoperatório}

A atuação de enfermagem neste período tão sensível ao paciente objetiva avaliar, detectar e intervir precocemente nas possíveis complicações. Além disso, contribui significativamente para a redução do risco de rejeição e aumento da qualidade de vida. Visto que esta fase demanda cuidados específicos da enfermagem, é importante a identificação precoce de diagnósticos com intervenção imediata nas possíveis situações emergentes ${ }^{9}$.

Cabe ao enfermeiro garantir a segurança do paciente, proporcionando uma cirurgia segura ao implantar protocolos e registros bem feitos. Além disso, atua assistindo ao paciente de forma integral e liderando a equipe de enfermagem ${ }^{8}$. 


\section{Pós-Operatório}

É o período mais importante para o transplantado, em que existem expectativas acompanhadas de ansiedade, já que há possibilidade de retornar a terapia dialítica ${ }^{9}$.

As primeiras 24 horas após o transplante renal são consideradas como período crítico, marcado pela necessidade de reposição parenteral de grande quantidade de líquidos, instabilidade hemodinâmica e respiratória, em que há grande chance do surgimento de complicações, como a rejeição ao enxerto. $\mathrm{O}$ enfermeiro atuante nesta etapa precisa ter conhecimento especializado para reduzir, prevenir ou antecipar a detecção dos problemas, bem como intervir o mais breve possível para intensificar as chances de sucesso do enxerto em longo prazo, e prestar assistência qualificada durante toda a internação. A SAE também está intrinsecamente inserida neste momento como instrumento indispensável para instituir as necessidades de cuidados identificadas e atingir os objetivos terapêuticos ${ }^{3,9}$.

A SAE presente em todas as fases da assistência é composta por cinco etapas: histórico, diagnóstico, planejamento, implementação e avaliação. O planejamento dos cuidados é reavaliado periodicamente de acordo com as necessidades individuais ${ }^{10}$. Outro instrumento de grande valor na assistência é o registro de enfermagem, que permite a comunicação com outros membros da equipe de saúde, disponibiliza dados para avaliação do estado geral, para implementação de assistência integral e holística, além disso, respalda legalmente o profissional, a instituição e o paciente ${ }^{3}$.

Existem diferenças na assistência de enfermagem no que diz respeito ao transplante inter vivos quando comparado ao rim recebido de um doador com morte encefálica e assistolia, com relação à função imediata do enxerto. $\mathrm{O}$ segundo caso apresenta maior incidência de falha do enxerto, e por isso demanda mais cuidados e monitorização ${ }^{3}$.

A diurese é o principal elemento de monitorização da função renal, pois é determinante de condutas terapêuticas como hidratação, medicação e da cirurgia com relação a complicações 
urológicas que envolvem a anastomose ureterovesical. A inserção de cateter vesical fornece medição precisa da saída de urina e determina a presença de hemorragia e coágulos sanguíneos, trombose vascular aguda e rejeição do órgão, além de ser fator preditor da evolução do transplante. A remoção do cateter é feita após cicatrização da anastomose do ureter na bexiga, fazendo necessária a continuação do monitoramento do débito urinário ${ }^{3}$.

A equipe de enfermagem deve ser bem treinada para a detecção precoce de sinais e sintomas de rejeição ou infecção, pois o sucesso do enxerto depende principalmente da qualidade dos cuidados prestados. A atuação do enfermeiro contribui inclusive para a redução do tempo de hospitalização ${ }^{8}$.

O paciente transplantado renal demanda uma maior carga de trabalho, que se não for cumprida e avaliada corretamente, pode levar a um prognóstico desfavorável. Comparados a pacientes de alta dependência, os pacientes em pós-operatório apresentam pontuação similar no Sistema de Classificação de Pacientes (SCP) ${ }^{11}$.

Após o procedimento cirúrgico são necessárias ações importantes como uso de imunossupressores e o acompanhamento ambulatorial. $\mathrm{O}$ enfermeiro implementa esta assistência, enfatizando a importância da continuidade do tratamento para o sucesso do enxerto 9.

Dentre as orientações para alta hospitalar o paciente deve ser orientado a controlar e monitorar diariamente seu peso, alimentação, ingesta hídrica e diurese. É importante verificar se o paciente entendeu completamente todas as orientações, pois isso será determinante para que não ocorram complicações ${ }^{9}$.

A adesão a recomendações para fazer uso correto das medicações, prevenção de infecção, automonitoramento de sinais de rejeição, alimentação saudável, exercícios físicos e proteção solar são fundamentais para o prognóstico de sobrevida do enxerto ${ }^{12}$. 
As mudanças comportamentais dos pacientes estão alicerçadas na educação desenvolvida pelo enfermeiro no ambiente hospitalar durante todas as etapas, porém os indivíduos saem ainda com fragilidades de adesão, conforme mostra o estudo de Pedroso et al. $(2019)^{12}$. Estas alterações na conduta do paciente, como foi discutido estão inter-relacionadas às orientações fornecidas pelo enfermeiro.

A sobrevida do enxerto depende, em grande escala, dos cuidados com a alimentação, já que o não cumprimento das orientações nutricionais pode acarretar obesidade, osteoporose, hiperlipidemia, hipertensão e diabetes, os quais são efeitos adversos das drogas imunossupressoras utilizadas por todos os pacientes transplantados ${ }^{12}$.

Também são fundamentais orientações acerca da higiene corporal e do domicílio, que tem como finalidade diminuir os riscos de infecção e rejeição do enxerto renal. No que tange às restrições estão o contato físico apenas com máscara de proteção facial, evitar aglomerações para impedir o risco de infecções, já que o regime imunossupressor induz susceptibilidade à infecção, neoplasias malignas e doença cardiovascular ${ }^{12}$.

Os estudos trouxeram como um dos principais cuidados no transplante renal a utilização de imunossupressores, que é feita com o objetivo de prevenir ou tratar a rejeição e de modo contínuo. Esta temática esteve em destaque nos estudos, como na pesquisa de Inácio et al. (2014) em que os profissionais citaram com maior frequência ${ }^{13}$.

Em pesquisa realizada por Albuquerque et al. (2010), o diagnóstico de fadiga se mostrou presente desde as sessões dialíticas dos pacientes, em razão da dieta restrita e locomoção ao serviço ${ }^{7}$. Houve também destaque do diagnóstico padrão de sono perturbado, em relação ao despertar a noite cerca de três vezes, que pode estar ligado ao aumento da ingesta hídrica uso de diuréticos. Outro estudo justificou este último diagnóstico com a preocupação financeira e aposentadoria precoce por invalidez, características presentes nos participantes da pesquisa, além do medo da rejeição e dos fatores apresentados pelo outro estudo ${ }^{14}$. 
Já o diagnóstico de nutrição desequilibrada, maior do que a necessidades corporais, apresentou como preditores o estado civil e a procedência, e estabelece relação com o aumento do apetite em virtude da imunossupressão, sedentarismo e retorno das condutas alimentares antes mais restritas ${ }^{7}$.

Referente à sexualidade, Lira et al. (2010) apresentaram o diagnóstico de disfunção sexual e padrões de sexualidade ineficazes, que se relacionam, o que é justificado por alguns medicamentos apresentarem efeito colateral que interfere no desempenho sexual ${ }^{14}$.

Também foi citado o diagnóstico de percepção sensorial perturbada, visual, com relação ao avançar da idade dos pacientes, em que o cristalino se torna menos flexível, em que se desenvolve a presbiopia, processo acelerado pelos medicamentos como corticoides $^{7,14}$.

É de extrema importância a abordagem da equipe de enfermagem de modo integral, a qual tem como foco a manutenção de todos os sistemas orgânicos e complicações, que por serem frequentes neste grupo específico demandam a necessidade de maior capacidade para conhecer, prevenir e tratar. Para isso a assistência deste grupo profissional precisa ser qualificada, já que este paciente é acompanhado por toda a vida ${ }^{5}$.

Um estudo demonstrou que dos profissionais participantes, apenas $4 \%$ do grupo respondeu corretamente todos os cuidados necessários ao paciente pós-transplante renal, seguidos de $20 \%$ que acertaram 7 a 9 respostas, já o restante e maioria acertou apenas de 1 a 3 $(60 \%)$ e 4 a $6(20 \%)$. Ao analisar sobre treinamento e capacitação o tema ainda não havia sido abordado pela instituição e só $15 \%$ passou por educação continuada, destes os $2 \%$ da pesquisa que acertaram todas as questões ${ }^{5}$. 


\section{CONCLUSÕES}

A doença renal crônico terminal se apresenta como problema de saúde pública em decorrência da alta prevalência a nível tanto nacional quanto internacional e reflete comprometimento da qualidade de vida dos portadores em relação à população em geral, principalmente com relação àqueles submetidos ao tratamento de hemodiálise.

São poucos os indivíduos submetidos ao transplante renal, em razão da alta demanda e baixa quantidade de doadores disponíveis. Também há dificuldade em relação ao sucesso do enxerto, visto que são necessários fatores compatíveis que se não presentes ocorre a rejeição do órgão recebido.

Para isso é importante que a enfermagem preste uma assistência qualificada durante todo o processo para que o paciente mantenha a adesão a todos os cuidados e terapia, o que irá corroborar com o sucesso e aumento da sobrevida do enxerto. A equipe de enfermagem está presente em todas as fases do procedimento e tem elevada importância na redução das taxas de complicações, atuando como educador, principalmente, do indivíduo.

\section{REFERÊNCIAS}

1. Souza, T. L., Trindade, T. R., de Mendonça, A. E., \& da Silva, R. A. (jun de 2016). Necessidades humanas básicas alteradas em pacientes pós-transplante renal: estudo transversal. Online brazj nurs, pp. 265 - 275.

2. Sociedade Brasileira de Nefrologia. (2018). SBN 2017 Censo. Congresso Brasileiro de Nefrologia. Rio de Janeiro.

3. Silva, A. E., Pontes, U. O., Genzini, T., do Prado, P. R., \& Amaral, T. L. (2014). Revisão integrativa sobre o papel do enfermeiro no pós-transplante renal. Cogitare enferm , 597 - 603. 
4. Santos, C. M., Kirchmaier, F. M., Silveira, W. J., \& Arreguy-Sena, C. (2015). Percepções de enfermeiros e clientes sobre cuidados de enfermagem no transplante de rim. Acta Paul Enferm, 337 - 343.

5. Primo, H. F., \& Hayakawa, L. Y. (2017). Conhecimento da equipe de enfermagem na assistência ao paciente em pós-operatório de transplante renal. Revista UNINGÁ Review , 1117.

6. Camara, J. d., Lima, P. Q., de Sousa, S. d., \& Paiva, S. d. (2016). Estratégias implementadas pelo enfermeiro para aprendizagem do transplantado renal em imunossupressão. Cienc Cuid Saude, 282 - 287.

7. Albuquerque, J. G., Lira, A. L., \& Lopes, M. V. (2010). Fatores preditivos de diagnósticos de enfermagem em pacientes submetidos ao transplante renal. Rev Bras Enferm, $98-103$.

8. Pedro, L. d., \& Batista, A. d. (2017). Assistência de enfermagem ao paciente submetido a transplante renal: uma revisão integrativa. Rev Enferm UFJF , 51 - 56.

9. Dâmaso, A. G., dos Santos, C. S., \& Bezerra, A. S. (2017). Assistência de enfermagem nos cuidados perioperatórios de pacientes em transplante renal. Ciências Biológicas e de Saúde Unit, $271-282$.

10. Goi, C., Meier, A. C., Salamoni, H., Zanella, M. C., \& Rosanelli, C. L. (2013). Sistematização da Assistência de Enfermagem a pacientes pós-transplante renal. XXI Seminário de Iniciação Científica. Porto Alegre.

11. Kochhann, D. S., \& Figueiredo, A. E. (2020). Enfermagem no transplane renal: comparação da demanda de cuidado entre escalas. Acta Paul Enferm , 1 - 8.

12. Pedroso, V. S., Thurow, M. R., de Medeiro, A. C., Scarton, J., Rodrigues, S. T., \& de Siqueira, H. C. (2019). Orientações do enfermeiro e mudanças no comportamento: caminho para sobrevivência do usuário transplantado renal. Rev Norte Mineira de Enferm , 92 - 102. 
13. Inácio, L. A., Montezeli, J. H., Sade, P. M., Caveião, C., \& Hey, A. P. (2014). Atuação do enfermeiro nas orientações de alta ao paciente pós-transplante renal. Rev Enferm UFSM , $323-331$.

14. Lira, A. L., \& Lopes, M. V. (2010). Pacientes transplantados renais: análise de associação dos diagnósticos de enfermagem. Rev Gaúcha Enferm , 108 - 114. 